\title{
Autoimmune Pancreatitis Accompanied by Cholecystitis, Periaortitis and Pseudotumors of the Liver
}

\author{
Hiroyuki Matsubayashi ${ }^{a}$ Hiroyoshi Furukawa ${ }^{b}$ \\ Katsuhiko Uesakac Keiko Sasaki ${ }^{\mathrm{d}}$ Hiroyuki Ono ${ }^{\mathrm{a}}$ \\ Ralph H. Hruban ${ }^{e}$ \\ Division of aEndoscopy, blmage Diagnosis, cHepatopancreaticobiliary Surgery and \\ dPathology, Shizuoka Cancer Center, Nagaizumi, Japan; eDepartment of \\ Pathology, The Sol Goldman Pancreatic Cancer Research Center, Johns Hopkins \\ Medical Institutions, Baltimore, Md., USA
}

\section{Key Words}

Autoimmune pancreatitis · Pseudotumor · Periaortitis · Diagnosis · IgG4

\begin{abstract}
A variety of extrapancreatic lesions have been associated with autoimmune pancreatitis (AIP), and these lesions can be difficult to diagnose. We report a patient referred to Shizuoka Cancer Center with the diagnosis of a possible biliary carcinoma with liver metastasis who was shown to have AIP accompanied by pseudotumors of liver. Clinical imaging revealed diffuse enlargement of the head of the pancreas with irregular narrowing of the main pancreatic duct and inferior common bile duct, multiple liver masses, mediastinal lymphadenopathy, and thickening of the wall of the gallbladder and abdominal aorta. Cytology and biopsy from the pancreaticobiliary tract was negative for malignancy. Serum carcinoembryonic antigen (CEA) and carbohydrate antigen (CA19-9) levels were in the normal range, but soluble interleukin 2 receptor (sIL2R), IgG4 and antinuclear antibody were abnormally high (sIL2R: 2,550 U/ml; lgG4: 764 mg/dl).

Corticosteroid therapy was effective and these abnormal findings all improved. This case demonstrates the clinical importance of AIP accompanied by other systemic disorders in the differential diagnosis of patients with a pancreatic mass lesion.
\end{abstract}

\section{Case Report}

In March 2006, a 62-year-old man was referred from another hospital with diagnosis of possible distal bile duct carcinoma with liver metastasis. The patient's chief complaint was jaundice. He denied abdominal pain, fever, fatigue, appetite loss, and weight loss. The patient's history was remarkable for 
hypertension, gout and diabetes mellitus, and he routinely took peroral medications for these disorders. He had bilateral, multiple renal cysts, but no history of renal dysfunction. His brother had died of pancreatic cancer, otherwise his family history was unremarkable. On admission, physical findings were significant only for jaundice. Blood tests included bilirubin $2.8 \mathrm{mg} / \mathrm{dl}$ (after biliary drain insertion at the previous hospital), GOT $51 \mathrm{U} / \mathrm{l}$, GPT $119 \mathrm{U} / \mathrm{l}$, alkaline phosphatase $972 \mathrm{U} / \mathrm{l}$, amylase $36 \mathrm{U} / \mathrm{l}$ (normal 43 $116 \mathrm{U} / \mathrm{l}$ ), BUN $13.3 \mathrm{mg} / \mathrm{dl}$, creatinine $1.02 \mathrm{mg} / \mathrm{dl}$, glucose $198 \mathrm{mg} / \mathrm{dl}$, HbAlc 8.8\% (normal 4.3-5.8\%), hemoglobin $11.6 \mathrm{~g} / \mathrm{dl}$, and eosinophils $23 \%$ (normal 0-6\%). Hepatitis B virus surface antigen and hepatitis $C$ virus antibody were both negative. High levels of serum immunoglobulin $\mathrm{G}(1,977 \mathrm{mg} / \mathrm{dl}$; normal $870-1,700 \mathrm{mg} / \mathrm{dl}$ ) were recognized, particularly IgG4 (764 mg/dl; normal 4.8-105 mg/dl). RA test, anti-SSA and anti-SSB were negative, but antinuclear antibody was positive. Serum tumor markers such as CEA and CA19-9 were in the normal range $(2.4 \mathrm{ng} / \mathrm{ml}$ and $24 \mathrm{U} / \mathrm{ml}$, respectively), but sIL2R $(2,550 \mathrm{U} / \mathrm{ml})$ was about five times as high as the upper limit of normal $(220-530 \mathrm{U} / \mathrm{ml})$.

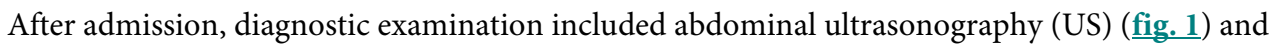
contrast-enhanced computer tomography (CT) (fig. 2) which revealed four nodular lesions in the liver (maximum $10 \mathrm{~mm}$ ), swelling of the pancreatic head and thickening of the wall of the gallbladder and abdominal aorta. T2-weighted SPIO-MRI showed multiple high-signal nodules in the liver. PET-CT revealed accumulation of FDG not only at the head of the pancreas, in the multiple liver masses, gallbladder and abdominal aorta, but also in multiple mediastinal lymph nodes. No obvious lymph node swelling was recognized around the abdominal aorta. ERCP demonstrated irregular narrowing of main pancreatic duct and lower common bile duct (fig. 3). With the combination of pancreatic head enlargement with narrowing of the main pancreatic duct and high serum levels of IgG, IgG4 and positive antinuclear antibody, the diagnostic criteria of AIP [1] were satisfied. To exclude cancer, we twice repeated biopsy (five sites each time) and brushing cytology from the narrowing site of the pancreaticobiliary duct. Tissue and fluid samples showed no evidence of an epithelial neoplasm and regenerating epithelial cells with abundance of inflammatory cells. IgG4-positive lymphocytes were sporadically recognized within the tissue (fig. 4). An endoscopic biliary drain was exchanged to prevent recurrence of the patient's jaundice. The clinical findings were compatible with AIP accompanied by mediastinal lymphadenopathy [1], pseudotumors of the liver [1-5], chronic cholecystitis, and periaortitis. Oral treatment with $40 \mathrm{mg}$ prednisolone was initiated and gradually decreased. Three months later, the imaging findings were all improved. The patients IgG, IgG4 and eosinophil count had also improved to $1,357 \mathrm{mg} / \mathrm{dl}, 399 \mathrm{mg} / \mathrm{dl}$, and $6.8 \%$, respectively. A year and ten months later, the patient was in healthy physical condition, had stopped steroid intake, and his serum creatinine level had improved from 1.25 to $1.02 \mathrm{mg} / \mathrm{dl}$, but he still had weak proteinuria. Serum glucose and HbA1c levels did not change significantly during the course of treatment.

\section{Discussion}

AIP is a systemic disease which, in addition to the pancreas, is known to affect multiple organs such as bile duct, cervical and abdominal lymph nodes, salivary gland, retroperitoneum, peripancreatic artery and vein $[1,6,7]$. Usually, this type of pancreatitis recovers structurally and functionally when the ductal obstruction is removed or the inflammation disappears after steroid administration [8]. According to a Japanese nationwide survey [9], the incidence of AIP was 0.82 per 100,000 people in 2002, with a higher ratio of males ( 2.85 times that of females). More than $95 \%$ of patients were past middle age ( $>45$ years). In this report, we present a patient with AIP with a variety of systemic disorders including mediastinal lymphadenopathy, hepatic pseudotumors, chronic cholecystitis, periaortitis, renal dysfunction and diabetes mellitus.

Because of the existence of multiple hepatic lesions, the possibility of pancreaticobiliary cancer with liver metastasis was considered. To date, four cases of AIP with pseudotumor of the liver, mimicking pancreaticobiliary carcinoma with liver metastasis, have been documented in the literature [2-5]. Several features helped establish the diagnosis in this case. First, the head of the pancreas was diffusely enlarged without a localized mass lesion. Second, the pancreatic duct was narrowed over a long length without an abrupt stenosis. These findings favored chronic inflammation in the head of the pancreas over carcinoma and were mentioned in the main condition of Japanese diagnostic criteria of AIP [1]. 
Serological data, such as high levels of serum IgG, IgG4 and positive antinuclear antibody, strongly supported the existence of IgG4-related autoimmune disease [7]. Hence, this case satisfied two of three conditions of diagnosis of the Japanese AIP criteria, i.e. clinical image and serum data, leading to the definitive diagnosis of AIP. Histocytology of tissue and fluid samples repeatedly obtained from the pancreaticobiliary tract, although nondiagnostic, may help exclude pancreaticobiliary cancer.

A broad differential diagnosis must be kept in mind when establishing a diagnosis in a patient with a mass-forming pancreatic lesion [10-13]. Time is of essence as many of these lesions grow rapidly [10-12] and the consequences of an accurate diagnosis are significant as therapy will vary greatly from high-dose chemotherapy to immunosuppression [1].

As for the hepatic lesions in our case, faint low echoic change by US and nondemarcated, low-density finding by enhanced CT supported the diagnosis of an inflammatory pseudotumor over that of metastastic carcinoma. Hepatic inflammatory pseudotumors sometimes show histology with IgG4-positive lymphoplasmacytic aggregation. Although no histological evidence was obtained from hepatic lesions, the therapeutic effect of corticosteroid [3,4] also supported the diagnosis of inflammatory pseudotumor.

We also noticed aortic wall thickness and renal dysfunction. AIP is known to be accompanied with obstructive phlebitis $[14,15]$ in small- to medium-sized veins, but not periaortitis. Similar to the hepatic lesion, we were not able to establish a definitive diagnosis of periaortitis with the histological evidence, because open biopsy of the abdominal wall is too invasive and in contrast, US and CT $[16,17]$ certainly support the diagnosis of chronic periaortitis [16]. Chronic periaortitis often accompanies peritoneal fibrosis, an extrahepatic lesion recognized in $10 \%$ of patients with AIP, and, like AIP, periaortitis is also caused by immune response to an antigen, only in the case an antigen localized to the aortic adventitia [18]. When periaortitis and retroperitoneal fibrosis are advanced, they can lead to aortic rupture and renal failure due to ureteric obstruction, so early detection is important. Immunosuppression with steroids or other agents is the preferred initial therapy, and if this is not effective, surgery is another option [16]. The patient we present also had renal dysfunction. As the patient did not have ureteric obstruction, other causes should be considered. For example, tubulointestinal nephritis is a recognized IgG4-related systemic disease [19]. Although we did not have periaortic or renal tissue to study, the efficacy of the steroid therapy suggests that periaortitis and renal diseases were a manifestation of this person's autoimmune disease. Clinicians should keep in mind that these findings may accompany AIP as part of a broader systemic autoimmune disorder.

Our case demonstrates the importance of clinical diagnosis using clinical imaging, blood tests and histological tests when AIP is associated with other systemic disorders. 
Fig. 1. Abdominal US. Multiple low-echoic nodules can be seen in the right lobe of the liver (a). The gallbladder wall is thickened (b). The pancreatic head is enlarged with low echoic change. The main pancreatic duct is slightly dilated in the body of the pancreas (c). The anterior side of aortic adventitia (arrows) is swollen (d).
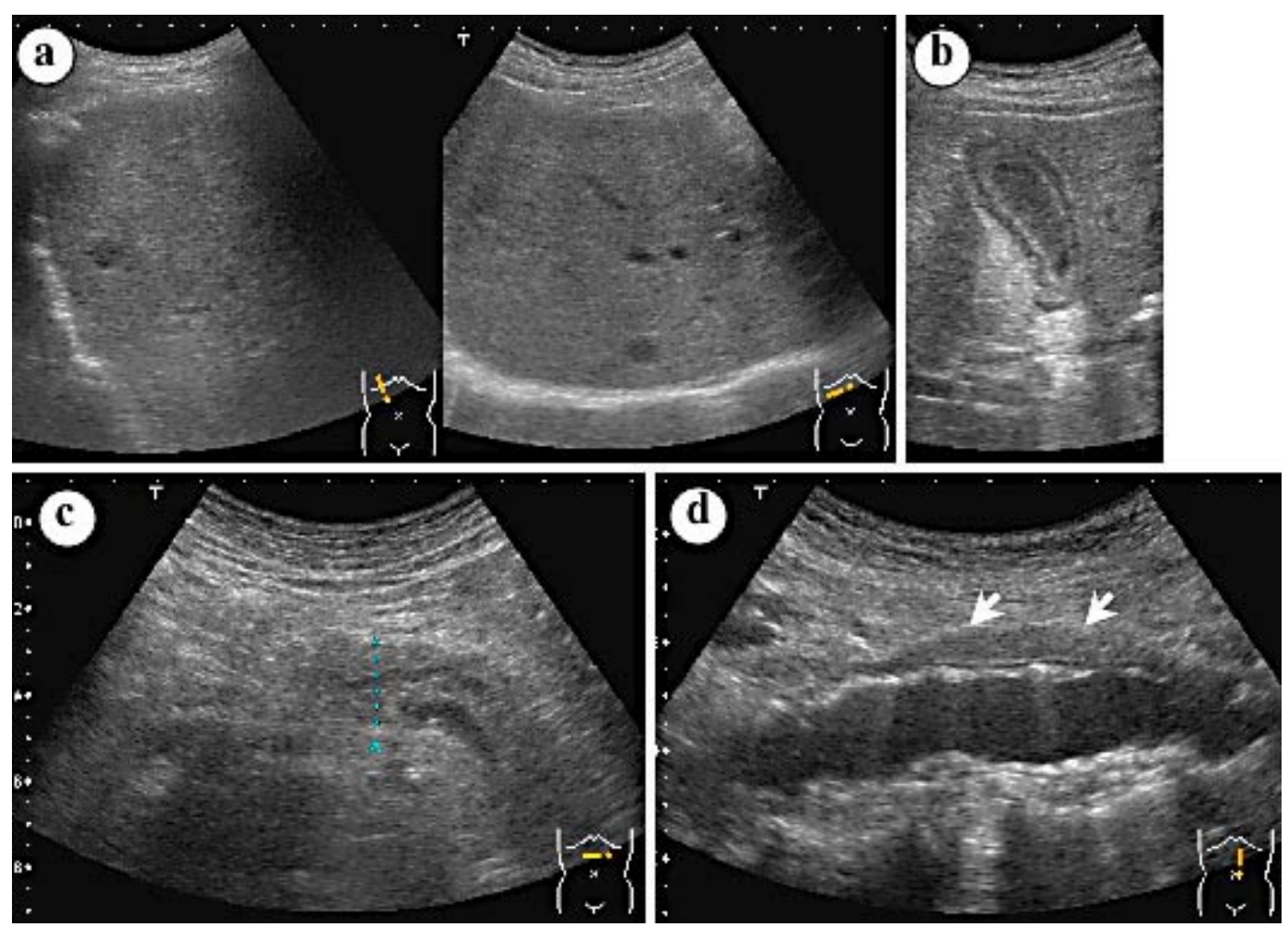
Fig. 2. Contrast-enhanced CT. Multiple low-density lesions were recognized in the right lobe of the liver $(\mathbf{a}, \mathbf{b})$. The wall of the gallbladder is thickened (c). The head of the pancreas (arrow) is enlarged with spotty low-density change. Biliary drain was inserted into the lower bile duct (d). The anterior wall of the abdominal aorta (arrow) is thickened (e), a finding which improved after steroid therapy (f).
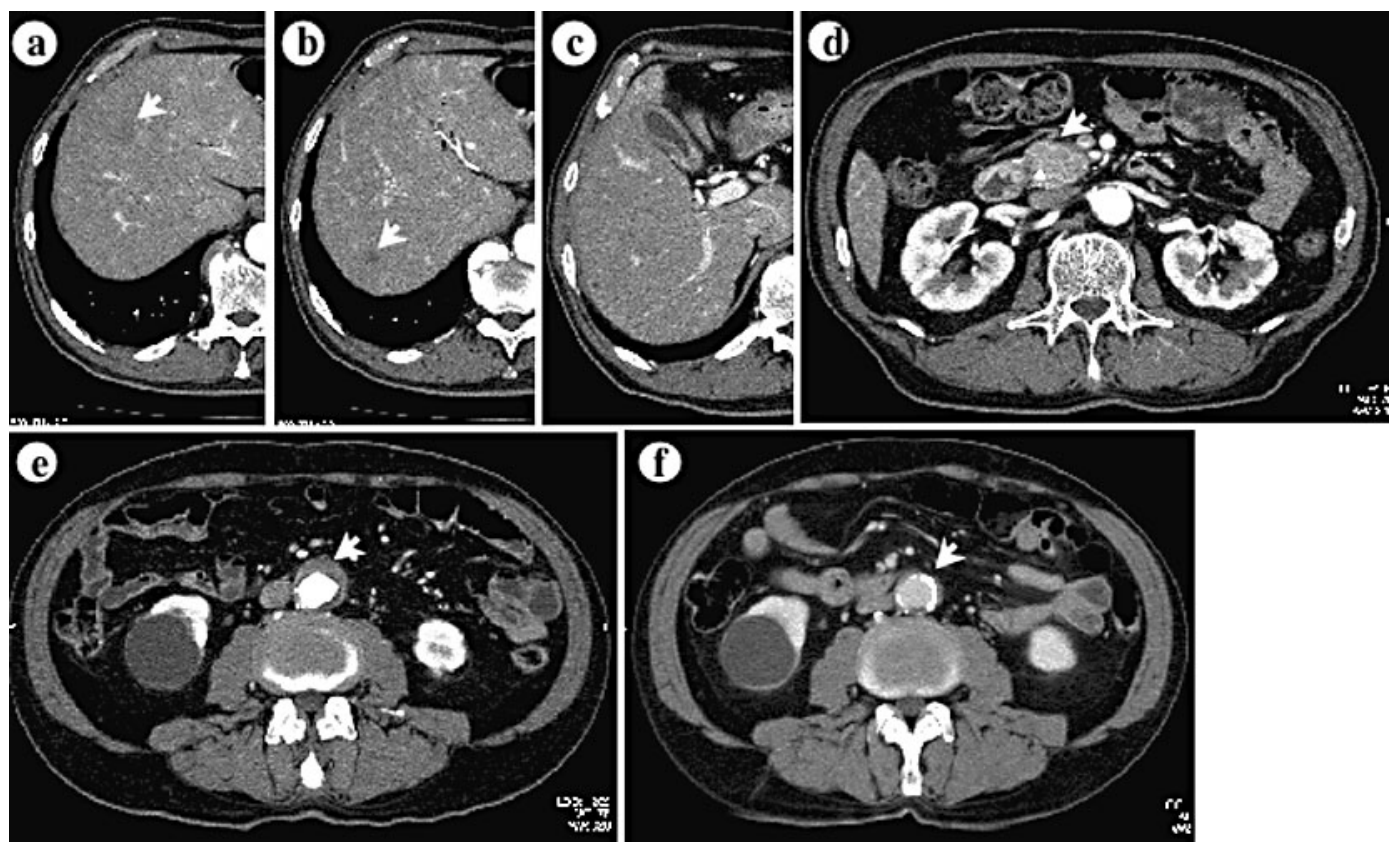

Fig. 3. Endoscopic retrograde cholangiopancreatography. A long segment of narrowing, without abrupt stenosis, was recognized in the main pancreatic duct at the pancreatic head (b) and lower bile duct (a).
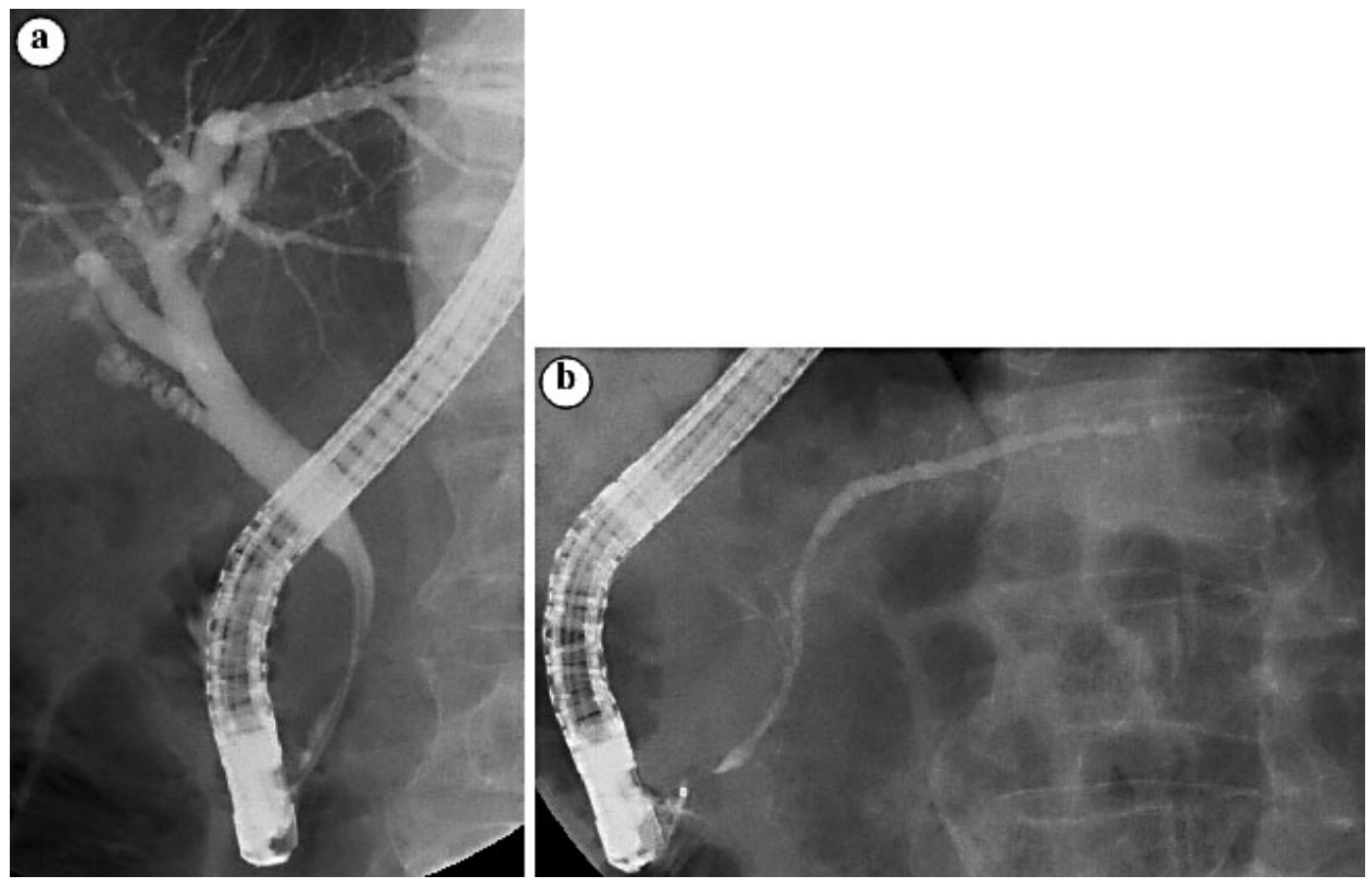


\begin{tabular}{r|l|l|l}
$\begin{array}{r}\text { Case Reports in } \\
\text { Gastroenterology }\end{array}$ & $\begin{array}{l}\text { Case Rep Gastroenterol 2008;2:155-161 } \\
\text { D0I: 10.1159/000125456 }\end{array}$ & Published online: May 24, 2008 & $\begin{array}{l}\text { O 2008 S. Karger AG, Basel } \\
\text { ISSN 1662-0631 } \\
\text { www.karger.com/crg }\end{array}$ \\
\hline
\end{tabular}

Fig. 4. Histological sample from the biliary duct at the stenotic site. Abundant inflammatory cells are associated with regenerating epithelial cells (a) (H\&E, $\times 200)$. IgG4-positive lymphocytes were sporadically recognized within the tissue (b) (IgG4 antibody, $\times 200)$.

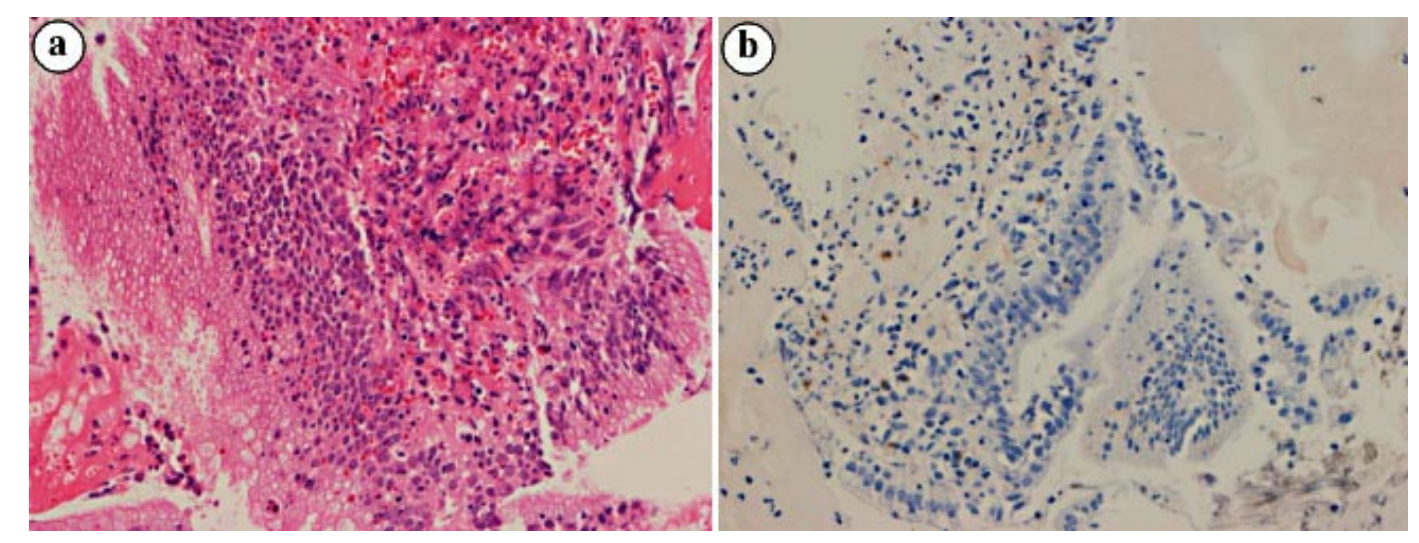




\section{References}

1 Okazaki K, Uchida K, Matsushita M, Takaoka M: How to diagnose autoimmune pancreatitis by the revised Japanese clinical criteria. J Gastroenterol 2007;42(suppl 18):32-38.

2 Sato Y, Harada K, Nakanuma Y: Hepatic inflammatory pseudotumour related to autoimmune pancreatitis. Histopathology 2004;45:418-419.

3 Sasahira N, Kawabe T, Nakamura A, Shimura K, Shimura H, Itobayashi E, Asada M, Shiratori Y, Omata M: Inflammatory pseudotumor of the liver and peripheral eosinophilia in autoimmune pancreatitis. World J Gastroenterol 2005;11:922-925.

4 Kanno A, Satoh K, Kimura K, Masamune A, Asakura T, Unno M, Matsuno S, Moriya T, Shimosegawa T: Autoimmune pancreatitis with hepatic inflammatory pseudotumor. Pancreas 2005;31:420-423.

5 Uchida K, Satoi S, Miyoshi H, Hachimine D, Ikeura T, Shimatani M, Matsushita M, Takaoka M, Takai S, Ashida K, Okazaki K: Inflammatory pseudotumors of the pancreas and liver with infiltration of IgG4-positive plasma cells. Intern Med 2007;46:1409-1412.

6 Kamisawa T, Egawa N, Nakajima H, Tsuruta K, Okamoto A: Extrapancreatic lesions in autoimmune pancreatitis. J Clin Gastroenterol 2005;39:904-907.

7 Kamisawa T, Nakajima H, Egawa N, Funata N, Tsuruta K, Okamoto A: IgG4related sclerosing disease incorporating sclerosing pancreatitis, cholangitis, sialadenitis and retroperitoneal fibrosis with lymphadenopathy. Pancreatology 2006;6:132-137.

8 Otsuki M: Chronic pancreatitis. The problems of diagnostic criteria. Pancreatology 2004;4:28-41.

9 Nishimori I, Tamakoshi A, Otsuki M: Prevalence of autoimmune pancreatitis in Japan from a nationwide survey in 2002. J Gastroenterol 2007;42(suppl 18):6-8.

10 Matsubayashi H, Seki T, Niki S, Mizumura Y, Taguchi Y, Moriyasu F, Go K: Wegener's granulomatosis with onset of acute pancreatitis and rapid progress. A case report. Pancreatology 2001;1:263-266.

11 Matsubayashi H, Fujiwara S, Kobayashi Y, Iiri T, Mitra A, Goggins M, Hruban RH, Moriyasu F: A small cell carcinoma of the pancreas with a high level of serum ProGRP. J Clin Gastroenterol 2004;38:834-835.

12 Matsubayashi H, Takagaki S, Otsubo T, Iiri T, Kobayashi Y, Yokota T, Shichijo K, Iwafuchi M, Kijima H: Pancreatic T-cell lymphoma with high level of soluble interleukin-2 receptor. J Gastroenterol 2002;37:863-867.

13 Matsubayashi H, Watanabe H, Ajioka Y, Nishikura K, Yamano M, Seki T, Saito T, Matsubayashi T: Different amounts of K-ras mutant epithelial cells in pancreatic carcinoma and mass-forming pancreatitis. Pancreas 2000;21:77-85.

14 Kamisawa T, Okamoto A: Autoimmune pancreatitis: proposal of IgG4-related sclerosing disease. J Gastroenterol 2006;41:613-625.

15 Suda K, Takase M, Fukumura Y, Ogura K, Ueda A, Matsuda T, Suzuki F: Histopathologic characteristics of autoimmune pancreatitis based on comparison with chronic pancreatitis. Pancreas 2005;30:355-358.

16 Jois RN, Gaffney K, Marshall T, Scott DG: Chronic periaortitis. Rheumatology (Oxford) 2004;43:1441-1446.

17 Iino M, Kuribayashi S, Imakita S, Takamiya M, Matsuo H, Ookita Y, Ando M, Ueda H: Sensitivity and specificity of CT in the diagnosis of inflammatory abdominal aortic aneurysms. J Comput Assist Tomogr 2002;26:1006-1012.

18 Rasmussen TE, Hallett JW Jr: Inflammatory aortic aneurysms. A clinical review with new perspectives in pathogenesis. Ann Surg 1997;225:155-164.

19 Saeki T, Saito A, Yamazaki H, Emura I, Imai N, Ueno M, Nishi S, Miyamura S, Gejyo F: Tubulointerstitial nephritis associated with IgG4-related systemic disease. Clin Exp Nephrol 2007;11:168-173. 